INPLASY

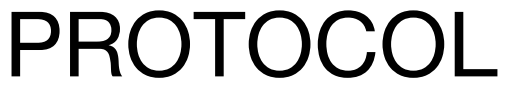

To cite: Yan et al. Robotic vs laparoscopic Roux-en-Y gastric bypass in morbidly obese patients: A systematic review and meta-analysis. Inplasy protocol 202090062. doi:

10.37766/inplasy2020.9.0062

Received: 14 September 2020

Published: 14 September 2020

Corresponding author: Long Yan

Izzy940@163.com

Author Affiliation:

Department of General

Surgery, 940th Hospital of Joint Logistics Support Force, Lanzhou 730050, Gansu

Province, China

Support: 2011GS04390.

Review Stage at time of this submission: Data analysis.

Conflicts of interest:

The authors have no conflicts of interest to disclose.

\section{Robotic vs laparoscopic Roux-en-Y gastric bypass in morbidly obese patients: A systematic review and meta-analysis}

\author{
Yan, L1; Fan, R²; Liu, H33 Xu, S4.
}

Review question / Objective: Robotic Roux-en-Y gastric bypass (RRYGB) and laparoscopic Roux-en-Y gastric bypass (LRYGB) are commonly performed bariatric procedures. The aim of this paper is to assess the outcomes and costs of these two kinds of surgical methods.

Condition being studied: Surgical management is the only long-term effective treatment to achieve meaningful and sustained weight loss. Roux-en-Y gastric bypass (RYGB) is one of the most commonly used procedure in all bariatric surgery. Some researchers claimed that robotic Roux-en-Y gastric bypass (RRYGB) has many advantages, such as RRYGB could specifically improve upon outcomes related to the gastrojejunal anastomosis (GJA) in RYGB, so they highly recommended RRYGB. However, others did not found that RRYGB has significant advantages over LRYGB.

INPLASY registration number: This protocol was registered with the International Platform of Registered Systematic Review and Meta-Analysis Protocols (INPLASY) on 14 September 2020 and was last updated on 14 September 2020 (registration number INPLASY202090062).

\section{INTRODUCTION}

Review question / Objective: Robotic Rouxen-Y gastric bypass (RRYGB) and laparoscopic Roux-en-Y gastric bypass
(LRYGB) are commonly performed bariatric procedures. The aim of this paper is to assess the outcomes and costs of these two kinds of surgical methods. 
Condition being studied: Surgical management is the only long-term effective treatment to achieve meaningful and sustained weight loss. Roux-en-Y gastric bypass (RYGB) is one of the most commonly used procedure in all bariatric surgery. Some researchers claimed that robotic Roux-en-Y gastric bypass (RRYGB) has many advantages, such as RRYGB could specifically improve upon outcomes related to the gastrojejunal anastomosis (GJA) in RYGB, so they highly recommended RRYGB. However, others did not found that RRYGB has significant advantages over LRYGB.

\section{METHODS}

Participant or population: Obese patients who adopted Robotic Roux-en-Y gastric bypass (RRYGB).

Intervention: Roux-en-Y gastric bypass.

Comparator: Laparoscopic Roux-en-Y gastric bypass.

Study designs to be included: We searched all of the studies till January 2020. The references of some relevant previous studies were also checked to make sure no eligible studies were missed. Search language was English, and search terms were: robotic, laparoscopic, Roux-en-Y gastric bypass, bariatric surgery.

Eligibility criteria: RCTs or nonRCTs comparing the outcomes and hospital charges of RRYGB and LRYGB in bariatric surgery were reviewed. Those selected studies included retrospective study and prospective study. And uncontrolled studies, surveys and case reports were excluded from the review.

Information sources: PubMed, Embase, Web of Science, Cochrane CENTRAL Register of Controlled Trials, Cochrane Database of Systematic Reviews. The references of some relevant previous studies were also checked to make sure no eligible studies were missed.
Main outcome(s): We mainly compared the outcomes and hospital charges between RRYGB and LRYGB. The contents of outcomes evaluation included surgical effect and surgical safety. The primary measure index for surgical effect was excess BMI loss (MIL) and the secondary measure index was BMI loss (\%BMIL). MIL $=$ (BMI baseline - BMI current) / (BMI baseline - BMI standard) ${ }^{*} 100 \% . \% B M I L=$ (BMI baseline - BMI current) / BMI baseline * $100 \%$. The measure indexes for surgical safety were incidence of intraoperative and postoperative complications. When we compared the hospital charges, we changed all of the hospitalization costs to US dollar at current exchange rates.

Quality assessment / Risk of bias analysis: We used the Newcastle-Ottawa Quality scale[7] to evaluate the quality of included nonRCTs (maximum points is 9) and Jadad score (maximum points is 5)[8] to evaluate the quality of included RCTs. The nonRCTs would be excluded from our meta-analysis if they had poor quality $(<5$ points). Publication biases were assessed with funnel plots.

Strategy of data synthesis: Based on an established brief plan, Two author independently extracted the relevant data from the selected studies with the tool of Microsoft Office Excel, including publication details, study characteristics, and the data of outcome assessment, hospital charges. And different opinions were submitted to the third author. It would be resolved by discussion until a consensus was made. All of the metaanalysis work was conducted by Review Manager 5, and we also used SPSS 20 to calculate and compare some means. All of the analysis work was carried out according to the Cochrane Handbook of Systematic Reviews of Interventions.

Subgroup analysis: We could adopt subgroup analysis on the basis of patients' gender, age, the date of surgery.

Sensibility analysis: Sensitivity analysis was made by adopting the fixed effects model and the random effects model at the same 
time during the meta-analyses, and then the differences of conclusions would be evaluated. If the fixed- and random-effects models produced the same result, only the fixed-effects estimate was reported. If the difference was significant, we would accept the result with fixed effects model when the 12 value $<50 \%$, and we would accept random-effects model when the 12 value $>=50 \%$.

Language: English.

Country(ies) involved: China.

Keywords: Obesity, Laparoscopic Roux-enY gastric bypass, Robotic Roux-en-Y gastric bypass, Bariatric surgery.

Contributions of each author:

Author 1 - Long Yan - Conceptualization, Data curation, Investigation, Methodology, Project administration, Writing - original draft.

Author 2 - Ruifang Fan - Conceptualization, Data curation, Methodology, Visualization, Writing - review \& editing.

Author 3 - Hongbin Liu - Funding acquisition, Supervision, Validation, Writing - review \& editing.

Author 4 - Shumei Xu - Data curation, Formal analysis. 